University of Wollongong

Research Online

Australian Institute for Innovative Materials -

Papers

Australian Institute for Innovative Materials

$1-1-2014$

Fabrication of free-standing hierarchical carbon nanofiber/graphene oxide/ polyaniline films for supercapacitors

\author{
Dongdong Xu \\ Zhengzhou University \\ Qun Xu \\ Zhengzhou University \\ Kaixi Wang \\ Zhengzhou University \\ Jun Chen \\ University of Wollongong, junc@uow.edu.au \\ Zhimin Chen \\ Zhengzhou University
}

Follow this and additional works at: https://ro.uow.edu.au/aiimpapers

Part of the Engineering Commons, and the Physical Sciences and Mathematics Commons 


\title{
Fabrication of free-standing hierarchical carbon nanofiber/graphene oxide/ polyaniline films for supercapacitors
}

\begin{abstract}
A hierarchical high-performance electrode with nanoacanthine-style polyaniline (PANI) deposited onto a carbon nanofiber/graphene oxide (CNF/GO) template was successfully prepared via an in situ polymerization process. The morphology analysis shows that introducing one-dimensional (1D) CNF could significantly decrease/inhibit the staking of laminated GO to form an open-porous CNF/GO architecture. Followed with in situ facial deposition of PANI, the as-synthesized PANI modified CNF/GO exhibits three-dimensional (3D) hierarchical layered nanoarchitecture, which favors the diffusion of the electrolyte ions into the inner region of active materials. The hierarchical free-standing electrodes were directly fabricated into sandwich structured supercapacitors using $1 \mathrm{M} \mathrm{H} 2 \mathrm{SO} 4$ as the electrolyte showing a significant specific capacitance of $450.2 \mathrm{~F} / \mathrm{g}$ at the voltage scan rate of $10 \mathrm{mV} / \mathrm{s}$. The electrochemical properties of the hierarchical structure can be further improved by a reduction procedure of $\mathrm{GO}$ before the deposition of PANI.
\end{abstract}

\section{Keywords}

free, fabrication, polyaniline, oxide, supercapacitors, graphene, films, nanofiber, carbon, hierarchical, standing

Disciplines

Engineering | Physical Sciences and Mathematics

\section{Publication Details}

Xu, D., Xu, Q., Wang, K., Chen, J. \& Chen, Z. (2014). Fabrication of free-standing hierarchical carbon nanofiber/graphene oxide/polyaniline films for supercapacitors. ACS Applied Materials and Interfaces, 6 (1), 200-209. 


\title{
Fabrication of Free-Standing Hierarchical Carbon Nanofiber/ Graphene Oxide/Polyaniline Films for Supercapacitors
}

\section{Dongdong Xu, ${ }^{\dagger}$ Qun Xu, ${ }^{*}, \boldsymbol{\dagger}$ Kaixi Wang, ${ }^{\dagger}$ Jun Chen, ${ }^{*}, \pm$ and Zhimin Chen ${ }^{\dagger}$}

\author{
${ }^{\dagger}$ College of Materials Science and Engineering, Zhengzhou University, Zhengzhou 450052, China

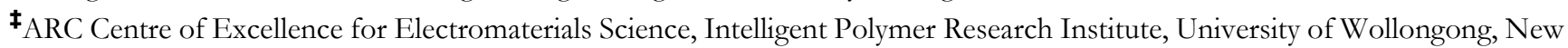 \\ South Wales 2522, Australia
}

\author{
* Supporting Information
}

ABSTRACT: A hierarchical high-performance electrode with nanoacanthine-style polyaniline (PANI) deposited onto a carbon nanofiber/graphene oxide (CNF/GO) template was successfully prepared via an in situ polymerization process. The morphology analysis shows that introducing one-dimensional (1D) CNF could significantly decrease/inhibit the staking of laminated GO to form an open-porous CNF/GO architecture. Followed with in situ facial deposition of PANI, the as-synthesized PANI modified CNF/GO exhibits three-dimensional (3D) hierarchical layered nanoarchitecture, which favors the diffusion of the electrolyte ions into the inner region of active materials. The hierarchical free-standing electrodes were directly fabricated into sandwich structured supercapacitors using $1 \mathrm{M} \mathrm{H}_{2} \mathrm{SO}_{4}$ as the electrolyte showing a significant specific capacitance of $450.2 \mathrm{~F} / \mathrm{g}$ at the voltage scan rate of $10 \mathrm{mV} / \mathrm{s}$. The electrochemical properties of the hierarchical structure can be further improved by a reduction procedure of GO before the deposition of PANI.

KEYWORDS: supercapacitors, carbon nanofiber, graphene oxide, three-dimensional, hybrid structure, free-standing film

\section{INTRODUCTION}

Supercapacitors as a new kind of electronic device are becoming much more multifunctional and developing in the trend of being lightweight, low cost, and flexible, in order to meet rapidly growing modern market demands. ${ }^{1-5}$ Depending on the charge-storage mechanism, they are basically classified into two types: electrochemical double-layer capacitors (EDLCs) based on carbon electrodes that store and release energy by nanoscopic charge separation at the electrochemical interface between electrode and electrolyte and pseudocapacitors with certain metal oxides or conducting polymers as the active electrode materials that associate with electrosorption and surface redox processes of the electroactive species. ${ }^{6-10}$ Generally speaking, EDLCs can achieve a long cycle life but with relatively low specific capacitance, while pseudocapacitors have much higher specific capacitance but poor cycle life that limits their real application in supercapacitors, ${ }^{11-13}$ due to the lack of suitable quality-designed supporting templates. Therefore, the combination of function-oriented nanocomposite electrodes using nanocarbon as the supporting materials to generate hierarchical architecture with both high performance and long cycle life is highly expected.

Nanostructured carbon materials are extensively used as electrode materials for supercapacitors (primarily EDLCs) because of their large surface areas and good conductivity, such as carbon fiber (CNF), carbon nanotube (CNT), graphene, and graphene oxide (GO). ${ }^{14-22}$ Among all the carbon materials, the electrochemical properties of one-dimensional
(1D) $\mathrm{CNF}$ and $\mathrm{CNT}$ are similar, and the $\mathrm{CNF}$ is more consistent with the requirement of a low cost compared to the CNT. Graphene-based electrodes, showing the potential to be a promising candidate for flexible supercapacitors, exhibit unsatisfactory capacitance performance due to the unavoidable aggregation of graphene and GO nanosheets. Therefore, the hybridization between different carbon materials is currently considered to be a feasible scheme to obtain the ideal electrode templates for supporting high-performance active materials. ${ }^{17-22}$ Conducting polymers have been considered as promising active electrode materials for the pseudocapacitors, owing to their low cost, easy synthesis, and high pseudocapacitance. $^{23,24}$ In particular, polyaniline (PANI) has been considered a key player due to its high theoretical specific pseudocapacitance according to its multiple redox states. Recently, several novel methodologies have been developed for the preparation of pure PANI and its composites. ${ }^{25-31}$ As most developed methods were reported in the preparation of powder-based PANI, binding materials became critical and must-needed electrodes. This might deteriorate the electrochemical properties of the as-prepared active electrodes. ${ }^{32-34}$ Therefore, the direct fabrication of free-standing polymer electrodes for capacitors has received considerable interest in recent years. ${ }^{5,35-38}$

\footnotetext{
Received: September 9, 2013

Accepted: December 10, 2013

Published: December 10, 2013
} 

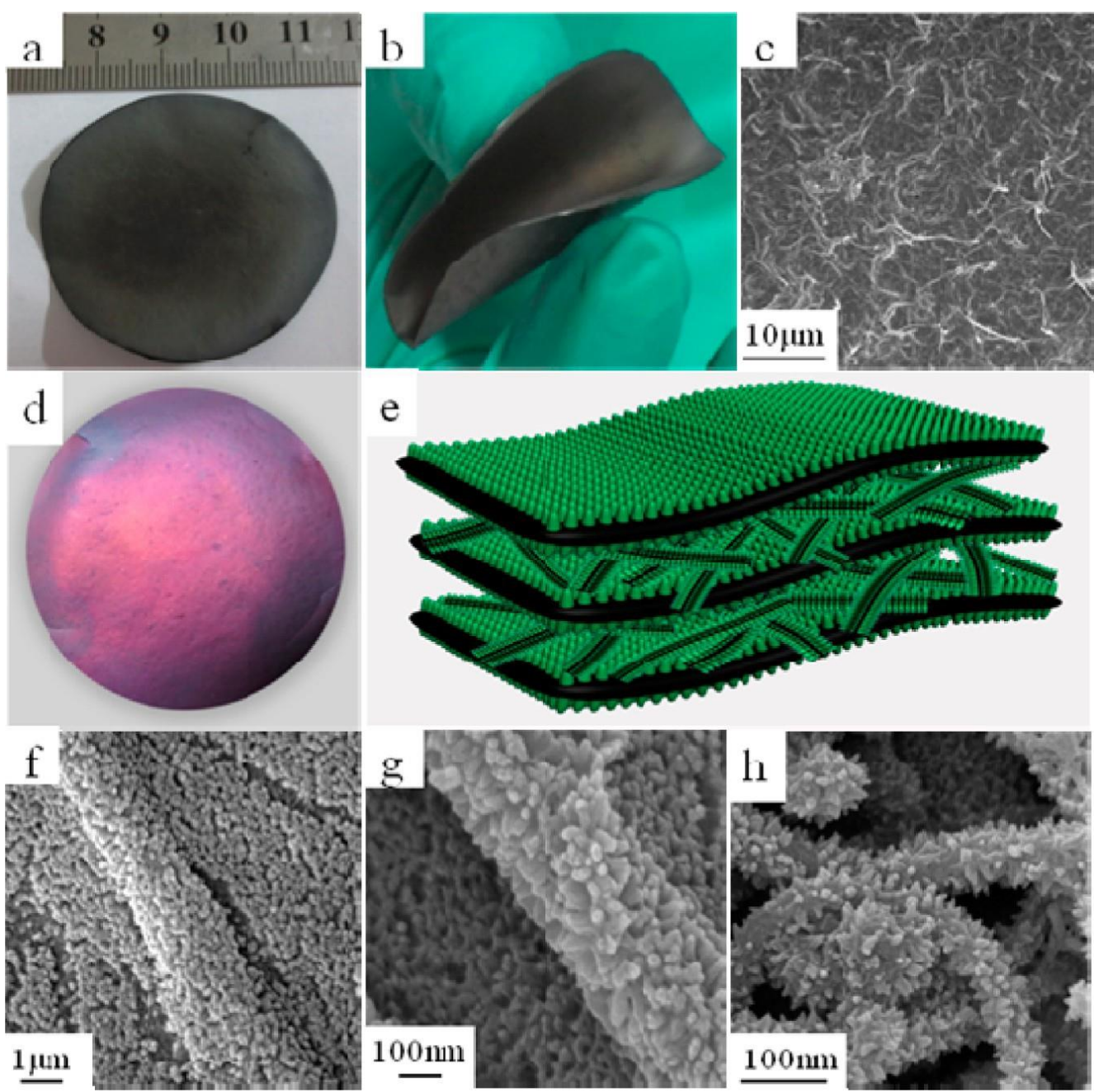

Figure 1. (a) Digital camera image of free-standing CNF/GO film. (b) A demo for the flexibility of the film. (c) Top-view SEM image of the CNF/ GO film. (d) Digital camera image of free-standing CNF/GO/PANI film prepared from the $C_{A N}$ of $30 \mathrm{mM}$. (e) A schematic illustration of the CNF/ GO/PANI film. (f and g) Top-view SEM images of CNF/GO/PANI at low and high magnification. (h) Side-view SEM image of CNF/GO/PANI film at a high magnification.

In this paper, we present the facial preparation approach to form the free-standing hierarchical PANI modified CNF/GO hybrid electrodes via in situ polymerization of aniline onto a preprepared porous $\mathrm{CNF} / \mathrm{GO}$ template in order to employ its advantages of good conductivity and flexibility. Specifically, $\mathrm{CNF}$ significantly reduced the aggregation degree of GO to form a porous structure and improve the utilization surface of GO greatly, because it acts as the reinforcing steel bar in the reinforced concrete structure of the $\mathrm{CNF} / \mathrm{GO}$ template. Followed with in situ facial deposition process, the resulting $\mathrm{PANI} / \mathrm{CNF} / \mathrm{GO}$ composite electrodes showed a unique threedimensional (3D) hierarchical microstructure with unique acanthine-style PANI nanowires covered on $\mathrm{CNF} / \mathrm{GO}$ supports. The high electrical conductivity and flexibility, in combination with the ideal form of hierarchical architecture, suggest that the as-prepared free-standing PANI/CNF/GO hybrid porous electrodes have the significant potential to be used as promising electrode materials for lightweight and flexible energy storage devices with stable high performance.

\section{EXPERIMENTAL SECTION}

2.1. Preparation of $\mathrm{CNF} / \mathrm{GO}$ and $\mathrm{CNF} / \mathrm{RGO}$ Hybrid Films. In a typical procedure, $15 \mathrm{mg}$ of CNFs (Pyrograf Products, Inc., USA) were dispersed into $1 \mathrm{wt} \%$ of sodium dodecyl sulfat (SDS) surfactant in $50 \mathrm{~mL}$ of distilled water. Simultaneously, the same amount of GO (see details in the Supporting Information) was also dispersed into 50 $\mathrm{mL}$ of distilled water in the absence of SDS. These solutions were then separately ultrasonicated for $2 \mathrm{~h}$ to form stable dispersions. The homogeneous solutions of $\mathrm{CNF}$ and GO were then mixed and ultrasonicated for another $0.5 \mathrm{~h}$, forming a black, homogeneous $\mathrm{CNF} /$ GO solution. To make a uniform film, this homogeneous dispersion was filtered through porous mixed cellulose membranes (pore size: $0.22 \mu \mathrm{m}$ ) with the aid of a vacuum. The resultant CNF/GO mat was then washed three times with $100 \mathrm{~mL}$ of distilled water to remove remaining surfactant. Subsequently, the dark $\mathrm{CNF} / \mathrm{GO}$ mat was allowed to dry overnight in a vacuum chamber at $50{ }^{\circ} \mathrm{C}$. The $\mathrm{CNF} /$ GO mat was then dipped in acetone solution for $4 \mathrm{~h}$ to remove the mixed cellulose membrane, and a free-standing CNF/GO film was obtained after drying. We introduce the use of the reducing agent, sodium borohydride $\left(\mathrm{NaBH}_{4}\right)$, in conducting the reduction process of GO. Typically, the as-prepared CNF/GO film was reduced by dipping it in an aqueous $\mathrm{NaBH}_{4}$ solution $(150 \mathrm{mM})$ for $2 \mathrm{~h}$. To remove excess $\mathrm{NaBH}_{4}$, the CNF/RGO film was washed with distilled water after reduction.

2.2. Preparation of CNF/GO/PANI Hybrid Films. CNF/GO/ PANI hybrid films were produced by in situ polymerization of aniline (AN). ${ }^{39}$ As-prepared CNF/GO films (30 mg) were immersed in a 70 $\mathrm{mL}$ aqueous solution containing aniline monomer and $1 \mathrm{M}$ perchloric 


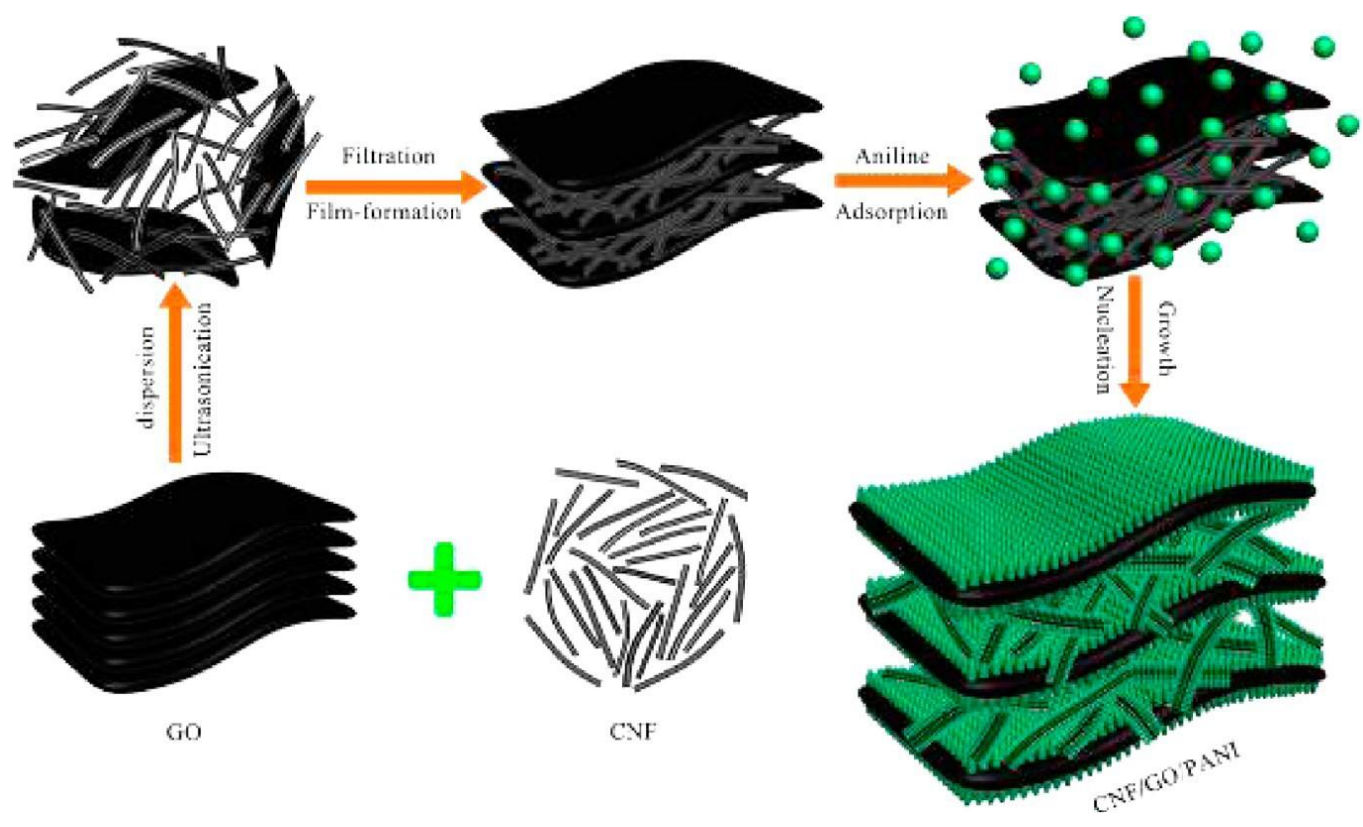

Figure 2. A formation mechanism of the CNF/GO/PANI film.

acid $\left(\mathrm{HClO}_{4}\right)$ for $30 \mathrm{~min}$, which would be helpful for the complete soakage of $\mathrm{CNF} / \mathrm{GO}$ in $\mathrm{HClO}_{4}$. One milliliter of ethanol was added into the reaction in order to avoid the solution being frozen. And then, another $30 \mathrm{~mL}$ of precooled $1 \mathrm{M} \mathrm{HClO}_{4}$ solution containing ammonium persulfate (APS) was added to the aniline solution through a drop-by-drop method. The molar ratio of AN/APS was 3:1. The polymerization was carried out under nitrogen in an ice-water bath with a temperature of $0-5{ }^{\circ} \mathrm{C}$. After polymerization for $7 \mathrm{~h}$, the $\mathrm{CNF} / \mathrm{GO} / \mathrm{PANI}$ hybrid films were taken out and washed with deionized water. Finally, the $\mathrm{CNF} / \mathrm{GO} / \mathrm{PANI}$ hybrid films were dried under vacuum conditions at $40{ }^{\circ} \mathrm{C}$ overnight. A series of $\mathrm{CNF} / \mathrm{GO} /$ PANI films were prepared from solutions with different aniline concentrations (CAN) of 10, 20, 30, 40, and $50 \mathrm{mM}$. The as-prepared CNF/GO/PANI films were marked as CGP-1, CGP-2, CGP-3, CGP4 , and CGP-5, respectively. The same method was used to obtain the $\mathrm{CNF} / \mathrm{RGO} / \mathrm{PANI}$ films. For comparison, pristine GO/PANI and pure PANI were also prepared at $30 \mathrm{mM}$ of aniline through the abovementioned process.

2.3. Characterization of As-Prepared Materials. The morphologies of the samples were analyzed by a field-emission scanning electron microscope (FE-SEM, JEOR JSM-6700F) and a transmission electron microscope (TEM; FEI Tecnai G2 20). Fourier transform infrared spectra (FTIR) of the products were recorded on a TENSOR 27 FTIR spectrometer (Bruker) in the absorption mode with a resolution of $2 \mathrm{~cm}^{-1}$. The Raman measurements were carried out on a Renishaw Microscope System RM2000 with a $50 \mathrm{~mW} \mathrm{Ar}{ }^{+}$laser at $514.5 \mathrm{~nm}$. X-ray photoelectron spectroscopy (XPS) measurements were made on a ESCALABMK spectrometer using electrostatic lens mode with a pass energy of $100 \mathrm{eV}$. $\mathrm{Al} \mathrm{K \alpha}$ radiation was used as the excitation source. Surface characterization of the materials was taken with an automatic $\mathrm{N}_{2}$ adsorption instrument (Micromeritics ASAP 2020 system).

2.4. Preparation of Supercapacitors Electrode and Electrochemical Performance Measurement. The electrochemical properties of the $\mathrm{CNF} / \mathrm{GO} / \mathrm{PANI}$ and GO/PANI hybrid films including cyclic voltammetry (CV), electrochemical impedance spectroscopy (EIS), and galvanostatic charge/discharge tests were measured with a two-electrode system in $1 \mathrm{M} \mathrm{H}_{2} \mathrm{SO}_{4}$ electrolyte at room temperature using a $\mathrm{CHI} 660 \mathrm{D}$ electrochemical working station. Specifically, the two-electrode sandwich-type cells were prepared by laying two pieces of active material with one piece of polypropylene (PP) film separator and two pieces of stainless steel mesh without using conductive additives and binders. The potential range for $\mathrm{CV}$ tests was 0 to $0.8 \mathrm{~V}$ at a series of scan rates varying from 5 to $100 \mathrm{mV} /$ s. EIS was performed at open circuit potential in the frequency range from $10^{4}$ to $0.1 \mathrm{~Hz}$ with the amplitude $5 \mathrm{mV}$. Galvanostatic charge/ discharge measurements were done from 0 to $0.8 \mathrm{~V}$ at different current densities. Cyclic stability was tested at a current density of $1 \mathrm{~A} / \mathrm{g}$ using a Land battery workstation (CT2001A, jinnuo electronic Co., Ltd., Wuhan).

\section{RESULTS AND DISCUSSION}

As shown in Figure 1a and b, the as-prepared free-standing $\mathrm{CNF} / \mathrm{GO}$ film is black and flexible. The surface morphology (Figure 1c) of the flexible $\mathrm{CNF} / \mathrm{GO}$ film depicts a uniform mat with typical interaction of the $\mathrm{CNF}$ and $\mathrm{GO}$ sheets. After the deposition of polyaniline (PANI), it shows a clear color change from black to dark purple (Figure 1d), which could be attributed to the modification of PANI as described in Figure 1e. Further characterization via SEM images (Figure 1f-h) indicates that the as-prepared CNF/GO/PANI has a lamellar microstructure with the acanthine PANI nanowires uniformly deposited onto the CNF/GO template to form a 3D hierarchical architecture. The structure of CNF/GO/PANI is further confirmed by the TEM image (Figure S1), and the length of the PANI nanowires can reach $50 \mathrm{~nm}$. Schematic preparation procedures of 3D CNF/GO/PANI architecture were illustrated in Figure 2. In the ultrasonication process, 1D CNFs were weaving in the layers of GO sheets with continuous and polydirectional combination. In general, GO sheets irreversibly aggregate and restack due to the strong $\Pi-\pi$ stacking and van der Waals force between the planar basal planes of GO sheets. ${ }^{40}$ While in the process of vacuum filtration, due to the interaction between CNFs and GO sheets, there exists a certain degree of inclination angle in the CNFs and GO sheets, which serves a mechanical supporting role to prevent the further accumulation of GO sheets. Finally, the reinforced concrete structure of $\mathrm{CNF} / \mathrm{GO}$ can be formed, and the microstructure orientation of the free-standing CNF/GO film is consistent and uniform. Followed with chemical deposition of PANI, the unique 3D hierarchical architecture CNF/GO/PANI was obtained. 

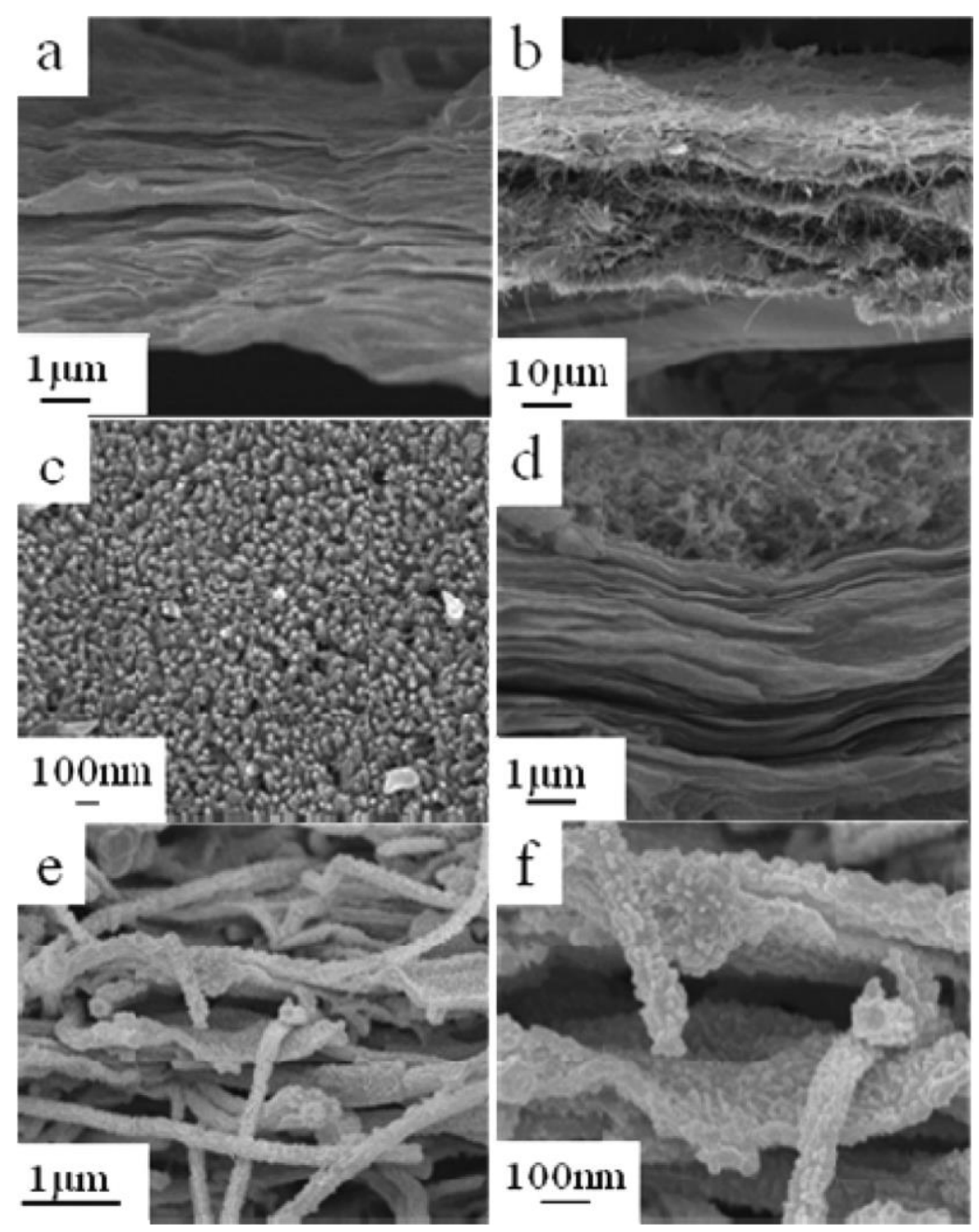

Figure 3. ( $a$ and b) Side-view SEM images of GO film and CNF/GO film, respectively. (c) Top-view SEM image of GO/PANI film. (d) Side-view SEM image of GO/PANI film. (e and f) Side-view SEM images of CNF/GO/PANI at low and high magnification.

A series of SEM images was characterized to further understand the preparation process of $3 \mathrm{D}$ hierarchical architecture, as shown in Figure 3. Figure 3a shows a crosssection view of the pure GO film; the fracture edge of the film reveals well-compact layer-by-layer stacking throughout the entire cross-section. The stacking of laminated GO is inhibited by introducing $1 \mathrm{D} \mathrm{CNF}$ to form a $\mathrm{CNF} / \mathrm{GO}$ hierarchical microstructure (Figure 3b), showing a clear stratification microstructure of $\mathrm{CNF} / \mathrm{GO}$. For the pure GO template, after the polymerization of PANI, the top surface of GO free-stand film (Figure 3c) was uniformly covered by a large quantity of small-compact PANI nanoparticles with a diameter of $30 \mathrm{~nm}$. However, the cross-section SEM image (Figure 3d) of GO/ PANI shows that no PANIs were observed in the inner layers of GO sheets. This phenomenon clearly suggests that for the pure GO film, due to the stacked lamellar structure, the polymerization of PANI only happened on the surface of GO film. In order to overcome the restacking issue of GO sheets to generate a porous 3D microstructure for PANI deposition, $\mathrm{CNF}$ s were introduced into the GO template, and the reinforced concrete structure of $\mathrm{CNF} / \mathrm{GO}$ was achieved. The hypothesis is verified by the cross-section views of the CNF/ GO/PANI (Figure 3e and f) showing an actual microstructure of the 3D hierarchical architecture completely in line with the model as described in Figure 2. The cross-section SEM images clearly demonstrate that the PANI has been successfully deposited onto a whole $\mathrm{CNF} / \mathrm{GO}$ template from the top surface, down to the interior of the architecture, including the individual CNFs.

The microstructures of deposited PANI hierarchical films were significantly influenced by the concentration of aniline monomer, which plays a key role during the polymerization of PANI, shown in Figure S2. At low concentration of aniline $\left(C_{\mathrm{AN}}=10 \mathrm{mM}\right)$, few PANI nanowires were observed, while the ordered long and vertical PANI nanowires were achieved at a $C_{\mathrm{AN}}$ of $30 \mathrm{mM}$. However, a further increase of $C_{\mathrm{AN}}$ led to the polymerized PANI nanowires inclined to merge together. Different from its surface morphology, the cross-section of the hierarchical film (Figure 4) exhibits an interesting change related to the increase of $C_{\mathrm{AN}}$. From the side views of the $\mathrm{CNF} /$ GO/PANI films (Figure 4a-h), which represent CGP-2, CGP3, CGP-4, and CGP-5, respectively, they all display a clear stratification in the hierarchical films. It can be seen that the $\mathrm{CNF}$ and GO layers are smooth and uncovered by PANI in the interior of the CGP-2 sample, which means the PANI just only exists on the surface of the film. The same experimental phenomena can be observed in CGP-1 as shown in Figure S3. As for the CGP-3, CGP-4, and CGP-5, we can clearly see the presence of PANI growth through the whole interior microstructure as shown in Figure $4 \mathrm{~d}, \mathrm{f}$, and h. Besides, the 


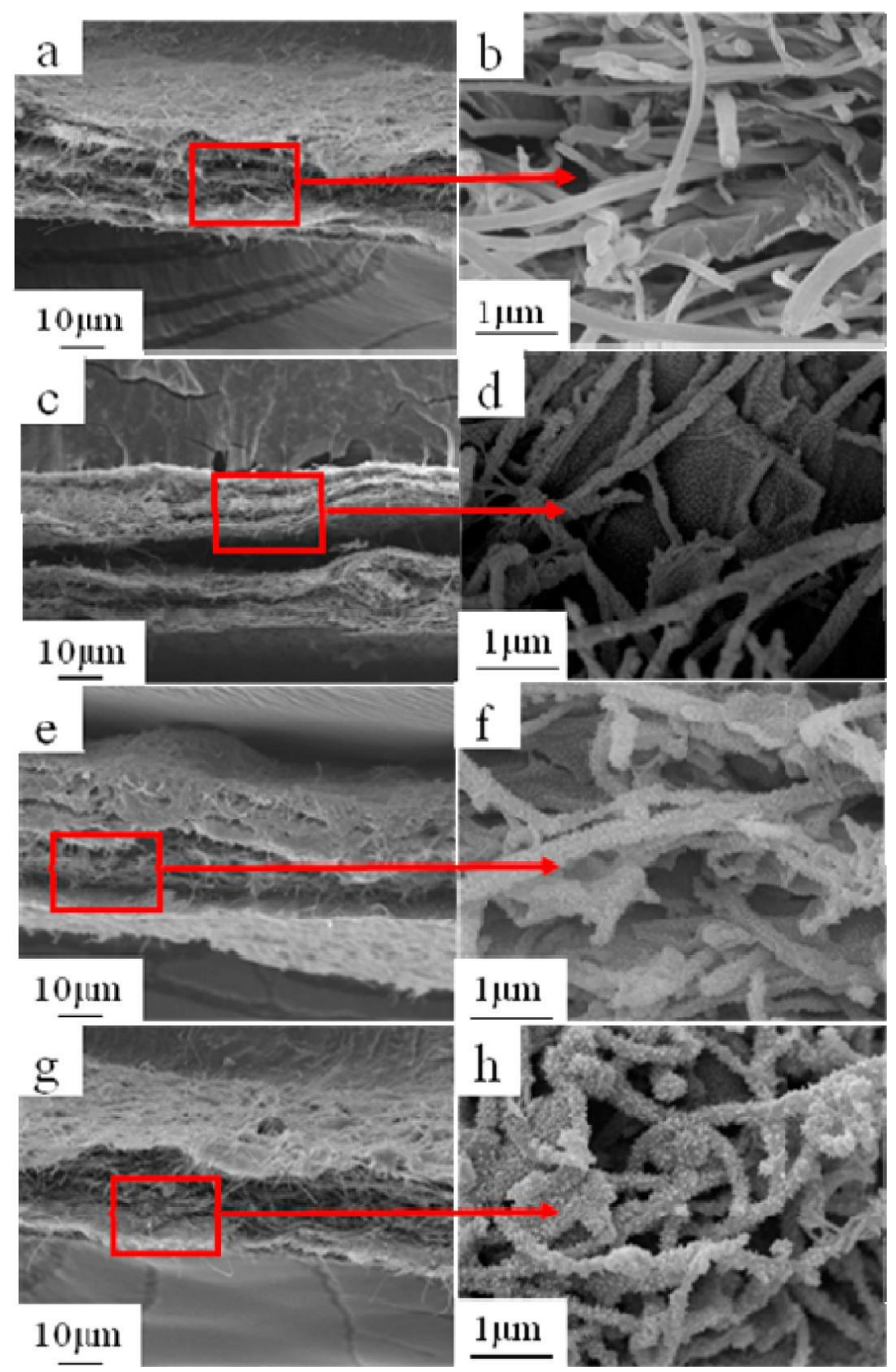

Figure 4. Side-view SEM images under different magnifications and different aniline concentrations of (a, b) $20 \mathrm{mM}$, (c, d) $30 \mathrm{mM}$, (e, f) $40 \mathrm{mM}$, (g, h) $50 \mathrm{mM}$.

growth situation of the interior PANI is similar to the PANI on the surface. Apparently, the acanthine structure of PANI protrudes more with the increase of aniline concentration, whether on CNFs or GOs.

The as-prepared composite architectures were further characterized by spectroscopy measurement. In the FTIR spectrum (Figure 5a) of CNF/GO, the peaks around 1730, 1635, and 1401 are attributed to the $\mathrm{C} \square \mathrm{O}$ in $\mathrm{COOH}$, intercalated water, and $\mathrm{C}-\mathrm{H}$ stretching, respectively. ${ }^{37,41}$ Compared with $\mathrm{CNF} / \mathrm{GO}$, the spectrum of CGP-3 displays two new peaks at 1570 and $1485 \mathrm{~cm}^{-1}$, which could be ascribed to the $\mathrm{C} \square \mathrm{C}$ stretching vibration of the quinoid ring and the benzene ring, ${ }^{42}$ indicating the presence of the oxidation state of PANI. The bands at 1308, 1242, 1120, and $800 \mathrm{~cm}^{-1}$ are corresponding to the $\mathrm{C}-\mathrm{N}$ stretching of the secondary aromatic amine, the $\mathrm{C}-\mathrm{N} \cdot{ }^{+}$stretching vibration in the polarized structure of PANI, and the aromatic $\mathrm{C}-\mathrm{H}$ bending in the plane and out of the plane for the aromatic ring, respectively. ${ }^{43}$

Raman spectroscopy provides a powerful tool to determine the microstructure of carbon-based materials and PANI polymer. Therefore, we performed Raman measurements on the $\mathrm{CNF} / \mathrm{GO}, \mathrm{PANI}$, and CGP-3 samples to further confirm the structure of PANI in the CGP-3 film. As shown in Figure $5 \mathrm{~b}$, the Raman spectrum of CNF/GO displays two prominent peaks at 1351 and $1580 \mathrm{~cm}^{-1}$, corresponding to the D-band (C-C, disordered graphite structure) and G-band $\left(\mathrm{sp}^{2}\right.$ hybridized carbon), respectively. ${ }^{44}$ For pure PANI and CGP3 samples, the bands at $1164,1219,1338,1482$, and $1590 \mathrm{~cm}^{-1}$ are assigned to in-plane $\mathrm{C}-\mathrm{H}$ bending of the quinoid ring, inplane $\mathrm{C}-\mathrm{H}$ bending of the benzenoid ring, the $\mathrm{C}-\mathrm{N}^{+}$ 

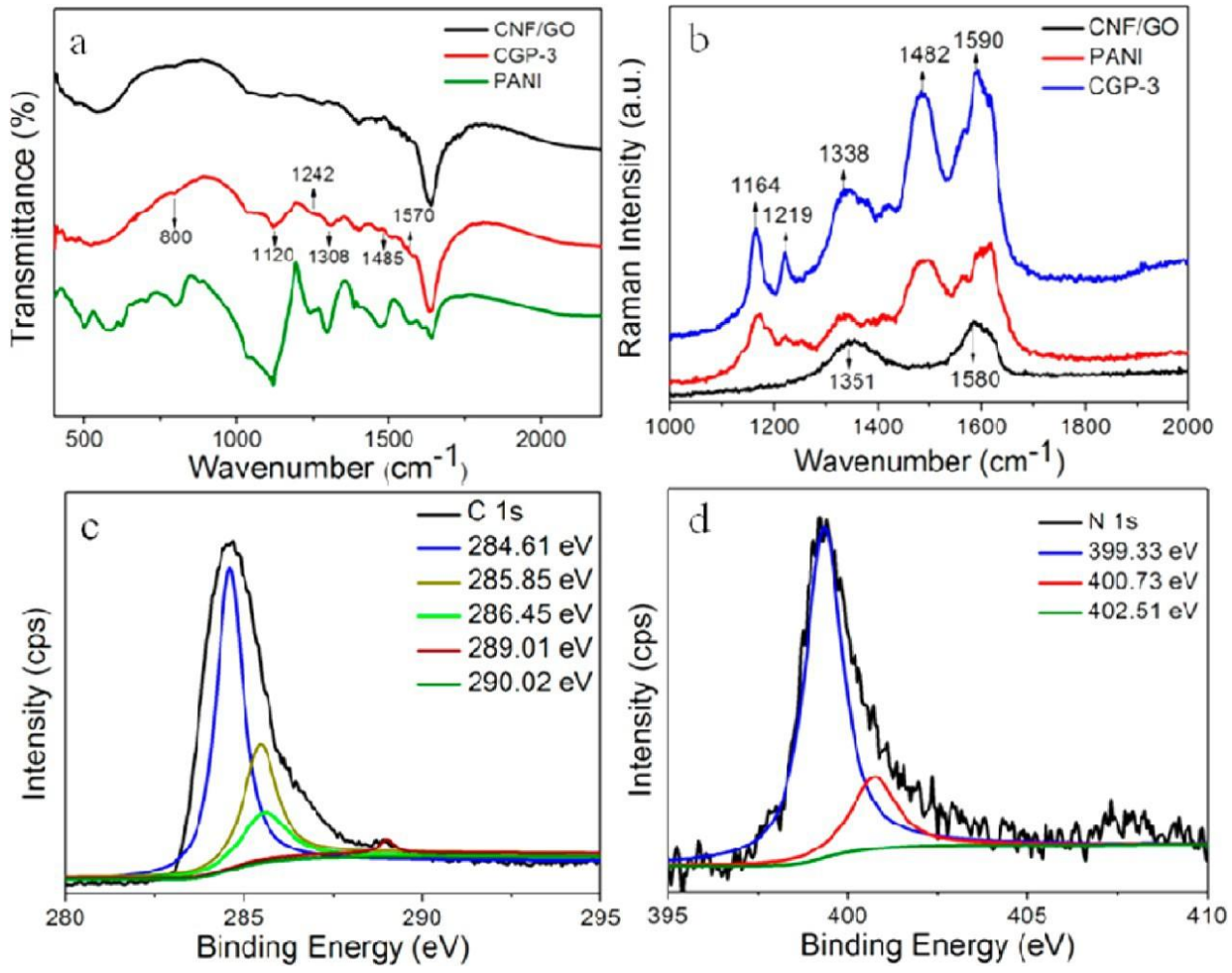

Figure 5. FTIR (a) and Raman (b) spectra of CNF/GO film, CNF/GO/PANI film, and PANI powder sample. C 1s (c) and N 1s (d) XP spectra for $\mathrm{CNF} / \mathrm{GO} / \mathrm{PANI}$.

stretching vibration, $\mathrm{C} \square \mathrm{C}$ stretching of the quinoid ring, and $\mathrm{C} \square \mathrm{C}$ stretching of the benzenoid ring, respectively, ${ }^{45,46}$ indicating the presence of a doped PANI structure.

XPS is used to investigate the different electronic structures of $\mathrm{CNF} / \mathrm{GO} / \mathrm{PANI}$. The $\mathrm{C} 1 \mathrm{~s}$ and the deconvolution of the $\mathrm{N}$ 1s core-level spectra of $\mathrm{CNF} / \mathrm{GO} / \mathrm{PANI}$ are presented in Figure $5 \mathrm{c}$ and $\mathrm{d}$, respectively. The $\mathrm{C} 1 \mathrm{~s}$ XPS spectrum is deconvoluted into five subpeaks, which are related to aromatic $\mathrm{C}-\mathrm{H}$ (284.61 eV), C-C/C-N/C $\square \mathrm{N} / \mathrm{C} \square \mathrm{N}^{+}(285.85 \mathrm{eV}$, $\mathrm{C}-\mathrm{O}$ (hydroxyl and epoxy, $286.45 \mathrm{eV}), \mathrm{O}-\mathrm{C}-\mathrm{O}(289.01 \mathrm{eV}$ ), and $\mathrm{O}-\mathrm{C} \square \mathrm{O}(290.02 \mathrm{eV}) .{ }^{25,47,48}$ The presence of $\mathrm{GO}$ and PANI is confirmed by the $\mathrm{O}-\mathrm{C} \square \mathrm{O}$ and $\mathrm{C} \square \mathrm{N} / \mathrm{C} \square \mathrm{N}^{+} / \mathrm{C}-$ $\mathrm{N}^{+}$, respectively. Moreover, the quinoid imine with Binding Energy (BE) at $399.33 \mathrm{eV}$, the benzenoid amine with $\mathrm{BE}$ centered at $400.73 \mathrm{eV}$, and the nitrogen cationic radical $\left(\mathrm{N}^{+}\right)$ with $\mathrm{BE}$ at $402.51 \mathrm{eV},{ }^{25,48}$ which are shown in the deconvolution of the $\mathrm{N}$ 1s core-level spectrum (Figure $5 \mathrm{~d}$ ), further suggest the presence of PANI.

To investigate the electrochemical performances of $\mathrm{CNF} /$ $\mathrm{GO} / \mathrm{PANI}$ in a real device, a symmetric supercapacitor device was constructed using $\mathrm{CNF} / \mathrm{GO} / \mathrm{PANI}$ as both an anode and a cathode. The cyclic voltammogram (CV; Figure 6a) of different $\mathrm{CNF} / \mathrm{GO} / \mathrm{PANI}$ samples were performed at a scan rate of 10 $\mathrm{mV} \mathrm{s}^{-1}$ and exhibit a typical redox behavior of PANI. Among all CNF/GO/PANI electrodes, the CGP-3 shows the highest specific capacitance of $450.2 \mathrm{~F} / \mathrm{g}$ (Table S1) according to the following equation.

$$
C_{\mathrm{s}}=2 \frac{\int I \mathrm{~d} V}{m \times \Delta V \times S}
$$

where $C_{\mathrm{s}}$ is the specific capacitance, $\int I \mathrm{~d} V$ is the integrated area of the CV curve, $m$ is the mass of the active materials of one electrode, $\Delta V$ is the voltage window, and $S$ is the scan rate. ${ }^{49}$

This phenomenon that the specific capacitance of CGP-3 is higher than the separate materials reported by Xu et al. ${ }^{50}$ could be possibly attributed to the internal microstructure of deposited PANI. It can be reflected from Figure S4, which is a relationship of $C_{\mathrm{s}}$ and PANI wt $\%$ toward $C_{\mathrm{AN}}$, that PANI wt $\%$ increases with an increase of $C_{\mathrm{AN}}$ at $C_{\mathrm{AN}}$ ranges from 10-50 $\mathrm{mM}$, while the $C_{\mathrm{s}}$ of $\mathrm{CNF} / \mathrm{GO} / \mathrm{PANI}$ increases with an increase of $C_{\mathrm{AN}}$ at low $C_{\mathrm{AN}}$ but decreases when the $C_{\mathrm{AN}}$ is higher than $30 \mathrm{mM}$. For the electrodes prepared from lower aniline concentration, such as CGP-1 and CGP-2, the PANI just exists on the surface of the film and cannot enter the inside of the hybrid film, so a less specific capacitance is inevitable. As for the CGP-4 and CGP-5, a large number of dissociated PANI nanowires covered the electrode, which can hinder the mass transfer and result in lower specific capacitance. The influence of aniline concentration on the capacitance property was further investigated by galvanostatic charge/discharge $(1 \mathrm{~A} / \mathrm{g})$. As shown in Figure 6b, the CGP-3 has the longest discharge time, which means the largest specific capacitance. This is consistent with the result of computing with CV.

To further investigate the advantages of hierarchical CNF/ GO/PANI as supercapacitor electrodes, the rate-dependent cyclic voltammograms of the CGP-3 electrode over a wide range of scan rates is shown in Figure 6c. It can be seen that the shape of $\mathrm{CV}$ was maintained even up to the high scan rate of $100 \mathrm{mV} \mathrm{s}^{-1}$, which indicates that the CGP-3 hybrid film has a rapid charge-discharge response within the potential window of $0.8 \mathrm{~V}$ in the device. In addition, Figure $6 \mathrm{~d}$ shows the comparison of electrochemical performance among $\mathrm{GO}, \mathrm{CNF} /$ $\mathrm{GO}, \mathrm{GO} / \mathrm{PANI}$, and $\mathrm{CNF} / \mathrm{GO} / \mathrm{PANI}$ films under identical 

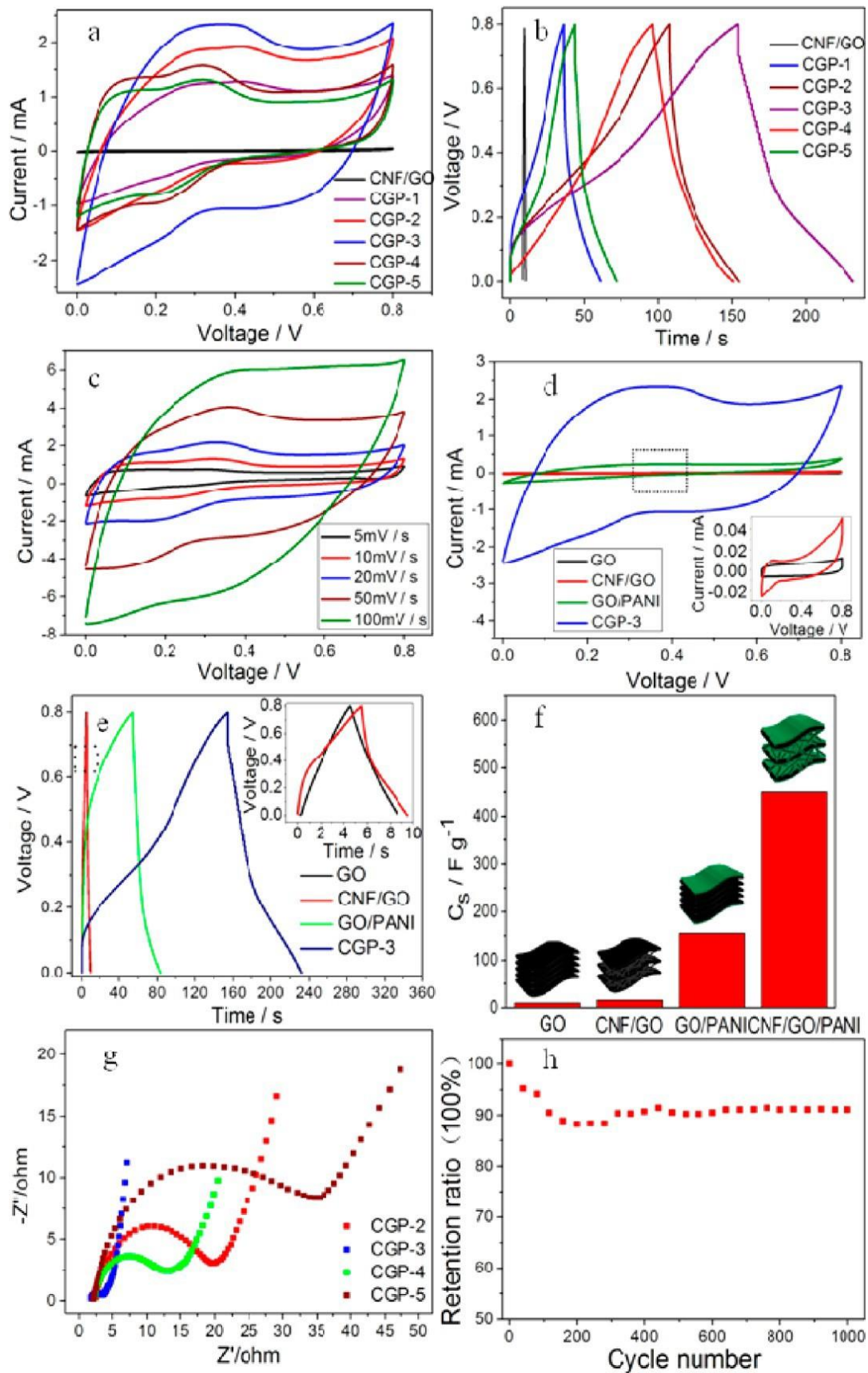

Figure 6. (a) CV curves of supercapacitors based on a CNF/GO/PANI hybrid film under different aniline concentrations, scan rate: $10 \mathrm{mV} / \mathrm{s}$. (b) Charge-discharge curves of CNF/GO/PANI hybrid film supercapacitors at different aniline concentrations, current density: $1 \mathrm{~A} / \mathrm{g}$. (c) CV curves of $\mathrm{CNF} / \mathrm{GO} / \mathrm{PANI}$ free-standing film supercapacitors at different scan rates. (d) CV of different film electrodes at $10 \mathrm{mV} / \mathrm{s}$. The inset depicts the enlarged view of the indicted square region. (e) Charge-discharge curves of different film electrodes at $1 \mathrm{~A} / \mathrm{g}$. The inset depicts the enlarged view of the indicted square region. (f) Relational graph of specific capacitance $\left(C_{\mathrm{s}}\right)$ to different films. The schematics representation of the microstructures of different films. (g) Nyquist impedance plots of the CNF/GO/PANI hybrid film supercapacitors with frequency ranging from $10 \mathrm{kHz}$ to $0.1 \mathrm{~Hz}$. (h) Plot of cycle life test of CGP-3 at a current density of $1 \mathrm{~A} / \mathrm{g}$.

conditions. The largest current and capacitance was obtained from CNF/GO/PANI. It is notable that the $\mathrm{CNF} / \mathrm{GO}$ exhibits no redox peak, although the presence of oxygen in GO should provide redox peaks due to the transition of the quinine/ hydroquionone form. ${ }^{37}$ Therefore, in order to understand the EDLC behavior or pseudocapacitive behavior of CNF/GO, a Bode plot has been provided in Figure S5, from which we can see that the phase angle of CNF/GO shows an obvious characteristic of a pseudocapacitor, which is between $0^{\circ}$ (the standard value of pure resistance) and $90^{\circ}$ (the standard value of physical capacitor). However, the pseudocapacitive behavior of CNF/GO is insufficient as compared with the CNF/GO/ PANI, and the outstanding performance of the latter can be attributed to two key reasons. First, the 3D acanthine PANI nanowire structure of CGP-3 provides numerous channels and shortens the diffusion lengths, which could benefit the ions' diffusion from solution to each PANI nanowire and ensures high utilization of the active material. Second, the tortuous 

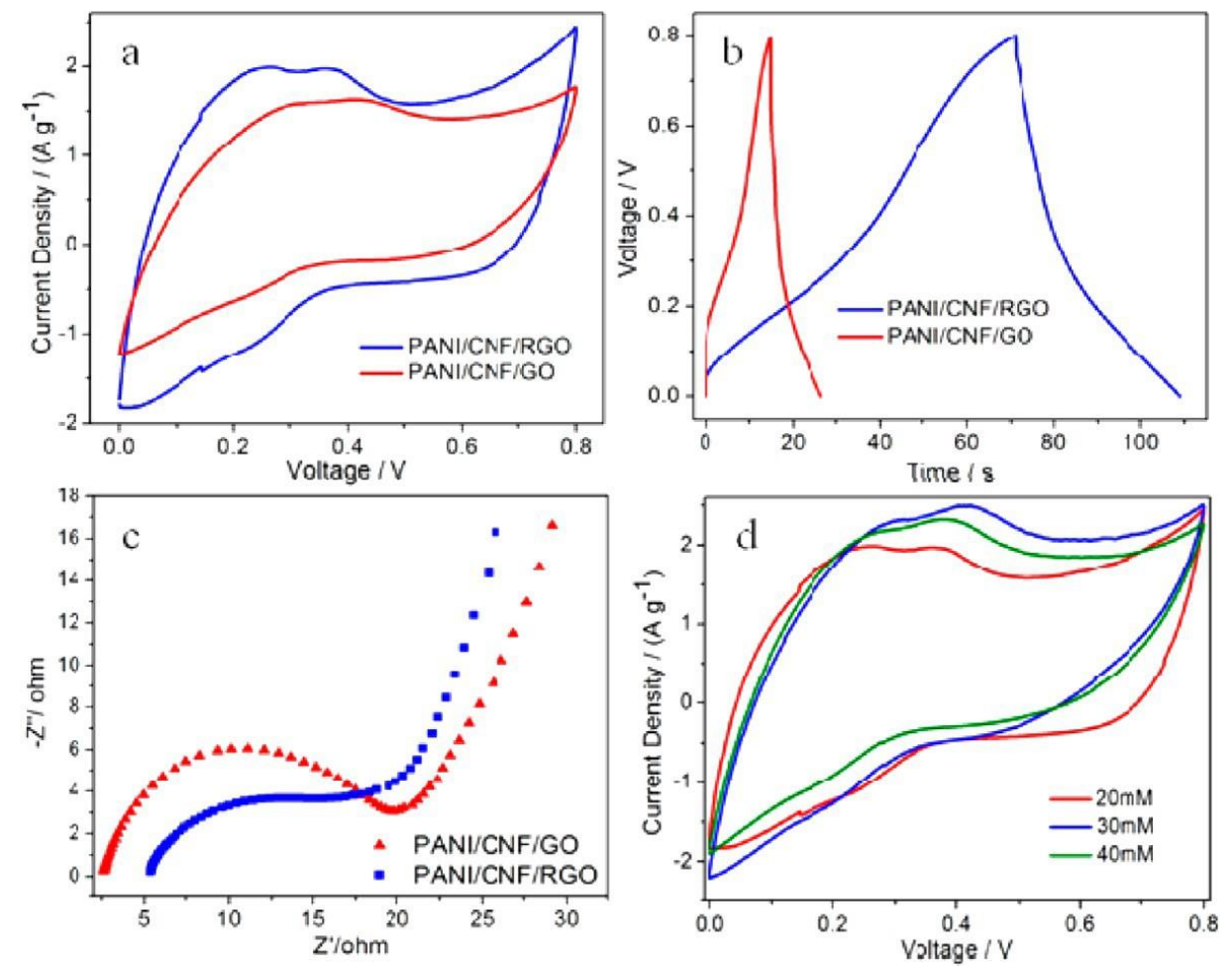

Figure 7. (a) CV curves of supercapacitors based on different hybrid film electrodes at $10 \mathrm{mV} / \mathrm{s}$. (b) Charge-discharge curves of different film electrodes at $2 \mathrm{~A} / \mathrm{g}$. (c) Nyquist impedance plots of different film supercapacitors with frequency ranging from $10 \mathrm{kHz}$ to $0.1 \mathrm{~Hz}$. (d) CV curves of supercapacitors based on CNF/RGO/PANI hybrid film under different aniline concentrations, scan rate: $10 \mathrm{mV} / \mathrm{s}$.

$\mathrm{CNF}$ functions as a conductive nanospacer not only reducing the aggregation of GO, which means a larger specific surface area for ions diffusion. The specific surface area of $\mathrm{CNF} / \mathrm{GO}$ was calculated on the basis of the Brunauer-Emmett-Teller (BET) theory is $26.1 \mathrm{~m}^{2} / \mathrm{g}$, which is higher than the pure CNF film $\left(11.4 \mathrm{~m}^{2} / \mathrm{g}\right) .^{38}$ Given that the specific surface area of GO film is very low, similar to the value of the chemically converted graphene film that has been reported by Shi et al., ${ }^{32}$ the introduction of the CNF dramatically increases the utilization of GO. Thus, the unique 3D hierarchical layered architecture plays a critical role in achieving high-level electrochemical performance in supercapacitor device. The same results can be analyzed with the charge/discharge performance, as shown in Figure 6e.

From the analyzing of Figure $6 \mathrm{~d}$ and e, a relational graph of specific capacitance (calculated from the above equation) vs different electrode materials is shown in Figure 6f. It can be concluded that the specific capacitances in the resulting supercapacitors using hierarchical CNF/GO/PANI as electrodes are 44 times that of GO film, 28 times that of bare CNF/ GO film, and 3 times that of the GO/PANI film, showing a significant improvement. On the other hand, as a plot of the imaginary component of the impedance against the real component, the EIS Nyquist plot demonstrates the frequency response of the electrode/electrolyte system. From the Nyquist plot shown in Figure $6 \mathrm{~g}$, it can be seen that at high frequencies the CGP-3 electrode displays the smallest semicircle, which is related to the lowest charge transfer resistance, and a transition to linearity at low frequency which exhibits an ideal capacitive behavior. ${ }^{40}$ Series internal resistance estimated from the intercept of the CGP-3 curve on the $x$ axis is about $1.7 \Omega$, which is lower than the similar system reported by Zhong et al., ${ }^{48}$ and according to the equivalent circuit, the charge transfer resistance was calculated to be $2.1 \Omega$, much less than the PANI/GECF-3 reported by He et al..$^{38}$ and the GO-PANI reported by Yan et al. ${ }^{37}$ Furthermore, from the magnified data in Figure S6a, a transition between the resistance capacitance semicircle and the migration of the electrolyte is observed at a frequency of about $26 \mathrm{HZ}$; the diffusion of electrolyte ions stopped at about $1 \mathrm{HZ}$, and thereafter the whole capacitance is reached. ${ }^{17}$ Figure S6b shows the dependence of phase angle on the frequency, which suggests the phase angle reaches $-45^{\circ}$ at a frequency of $0.3 \mathrm{~Hz}\left(f_{0}\right)$, giving a corresponding time constant $\left(\mathrm{T}_{0}\right)$ of $3.33 \mathrm{~s}$ for the device. ${ }^{1,50}$

Cycle life tests of CGP-3 are conducted at a current density of $1 \mathrm{~A} / \mathrm{g}$. As shown in Figure 6h, the capacitance retention of the electrode still keeps $90.2 \%$ of its original value after 1000 cycle tests. Usually, the degradation in capacitance is ascribed to swelling and shrinkage, which induced gradually deterioration of the conductivity and volumetric changes. ${ }^{53}$ While in our system, the hierarchical 3D structure of CNF/GO/PANI films with the high surface area holds enough void space to accommodate the volumetric change; therefore, the hybrid films exhibit a good stability and comparatively long lifetime as the supercapacitor.

As the electrical performance of $\mathrm{GO}$ can be improved through a reduction process, we further optimize the hybrid film by a reduction of GO using sodium borohydride $\left(\mathrm{NaBH}_{4}\right) ; 47$ after the deposition of PANI, the resulting hierarchical PANI/CNF/RGO film shows better electrochemical performance. The loss of oxygen-containing groups after reduction was confirmed by FTIR spectrum. As shown in Figure S7a, the intensities of peaks are smoother than CNF/ GO, and the new peaks arising from PANI at 1230, 1340, 1531 $\mathrm{cm}^{-1}$ indicate the generation of a PANI/CNF/RGO composite. ${ }^{42,43}$ In combination with FTIR spectroscopy, 
Raman scattering was measured to provide more information about the microstructure of PANI/CNF/RGO. From Figure $\mathrm{S} 7 \mathrm{~b}$, it is clearly observed that significant structural changes occur during the reduction processing from $\mathrm{CNF} / \mathrm{GO}$ to $\mathrm{CNF} /$ $\mathrm{RGO}$, and this observation is consistent with the general belief that defects decrease during the reduction process by the removal of oxygen atoms followed by the formation of double bonds, which means a higher graphitization degree of $\mathrm{CNF}$ / RGO. Similar to the PANI/CNF/GO, for the Raman spectrum of the PANI/CNF/RGO sample, the new peaks arise from PANI at 1162,1218 , and $1488 \mathrm{~cm}^{-1},{ }^{45,46}$ apart from the D and $\mathrm{G}$ bands of the CNF/RGO sample. Compared to PANI/CNF/ $\mathrm{GO}$, the decrease of the heterocarbon component in the $\mathrm{C} 1 \mathrm{~s}$ peak, such as that arising from hydroxyl (C-O) and epoxy (C$\mathrm{O}-\mathrm{C}$ ) groups $^{47}$ (Figure $\mathrm{S} 7 \mathrm{c}$ ), is consistent with the increased ratio of the $\mathrm{C}$ to $\mathrm{O}$ molecule from 1.5 to 1.9 in the PANI/ $\mathrm{CNF} / \mathrm{RGO}$ film. In addition, the similar deconvolution of the $\mathrm{N}$ 1s core-level spectrum (Figure S7d) indictes the presence of PANI molecular chains. ${ }^{48}$

The contrast figures of electrical properties of PANI/CNF/ $\mathrm{GO}$ and $\mathrm{PANI} / \mathrm{CNF} / \mathrm{RGO}$ samples (the $C_{\mathrm{AN}}$ of them are 20 $\mathrm{mM}$ ) were shown in Figure $7 \mathrm{a}-\mathrm{c}$. The CV curves (Figure $7 \mathrm{a}$ ) of the two samples were performed at a scan rate of $10 \mathrm{mV} \mathrm{s}^{-1}$; it is obvious that the current density response of $\mathrm{PANI} / \mathrm{CNF} /$ $\mathrm{RGO}$ is much larger than that of the PANI/CNF/GO film, indicating that the specific capacitance of the former is apparently larger than that of the latter. A consistent result can also be obtained from the curves of galvanostatic charge/ discharge, as shown in Figure 7b. It is notable that the IR drop of the PANI/CNF/RGO electrode is much lower than that of $\mathrm{PANI} / \mathrm{CNF} / \mathrm{GO}$ (Figure 6e), even if the former is under a higher current density, which shows that the materials can be optimized to decrease their internal resistances by the reduction of graphene oxide. From the Nyquist plot shown in Figure 7c, the Warbug-type line is illustrated (the slope of the $45^{\circ}$ portion of the Nyquist lots), the length of which can be used to characterize the ion diffusion process. So for the PANI/CNF/ RGO electrode, it is is much shorter than that of PANI/CNF/ GO. This demonstrates the fast ion transfer in the optimized electrode material. ${ }^{12,25}$ Further, the optimal $C_{\mathrm{AN}}$ for the PANI/ $\mathrm{CNF} / \mathrm{RGO}$ electrode is also $30 \mathrm{mM}$, which can be observed from Figure 7d, the specific capacitance of the different PANI/ CNF/RGO electrodes are 436, 479, and $430 \mathrm{~F} \mathrm{~g}^{-1}$, corresponding to $C_{\mathrm{AN}}$ 's of 20,30 , and $40 \mathrm{mM}$, respectively.

\section{CONCLUSIONS}

In summary, we have demonstrated that the free-standing hierarchical $\mathrm{CNF} / \mathrm{GO} / \mathrm{PANI}$ films can be delicately synthesized by in situ polymerization of aniline on the peculiar substrate, the reinforced concrete structure of CNF/GO film. The experimental result is a proof of concept that the ideal electrode templates for supporting high-performance active materials are decisive for excellent electrochemical properties. The calculated specific capacitances in the resulting supercapacitors using the peculiar hierarchical CNF/GO/PANI as electrodes are 44 times that of pure GO film, 28 times that of bare CNF/GO film, and 3 times that of the GO/PANI film, showing a significant improvement of the specific capacitance. Furthermore, the electrochemical properties of the hierarchical film can be further improved by a reduction procedure of GO. So this study supplies a promising and efficient way to obtain ideal electrode materials for lightweight and flexible energy storage devices with high performance.

\section{ASSOCIATED CONTENT}

Supporting Information

The preparation of GO. The TEM and SEM images of CNF/ GO/PANI hybrid film. Relational graph and table of specific capacitance (Cs) and content of PANI (PANI wt \%) to aniline concentration $\left(\mathrm{C}_{\mathrm{AN}}\right)$. The phase-frequency plot of $\mathrm{CNF} / \mathrm{GO}$, Nyquist plot and phase-frequency plot of CGP-3. FTIR, Raman and XPS spectra of PANI/CNF/RGO film.

\section{AUTHOR INFORMATION}

\section{Corresponding Author}

*Tel.: +86 371 67767827. Fax: +86 371 677678. E-mail: qunxu@zzu.edu.cn; junc@uow.edu.au.

Notes

The authors declare no competing financial interest.

\section{ACKNOWLEDGMENTS}

We are grateful for the National Natural Science Foundation of China (No. 51173170, 50955010, and 20974102), the financial support from the Innovation Talents Award of Henan Province (114200510019) and the Program for New Century Excellent Talents in University (NCET).

\section{REFERENCES}

(1) EI-Kady, M. F.; Strong, V.; Dubin, S.; Kaner, R. B. Science 2012, 335, 1326-1330.

(2) Simon, P.; Gogotsi, Y. Nat. Mater. 2008, 7, 845-854.

(3) Niu, Z. Q.; Zhou, W. Y.; Chen, J.; Feng, G. X.; Li, H.; Ma, W. J.; Li, J.Z.; Dong, H. B.; Ren, Y.;Zhao, D.; Xie, S. S. Energy Environ. Sci. 2011, 4, 1440-1446.

(4) Kaempgen, M.; Chan, C. K.; Ma, J.; Cui, Y.; Gruner, G. Nano Lett. 2009, 9, 1872-1876.

(5) Meng, C. Z.; Liu, C. H.; Chen, L. Z.; Hu, C. H.; Fan, S. S. Nano Lett. 2010, 10, 4025-4031.

(6) Yunseok, J.; Jeongdai, J.; Young, M. C.; Inyoung, K.; SeungHyun, L.; Daewon, K.; Seong, M. Y. Electrochim. Acta 2013, 102, 240245.

(7) Meryl, D. S.; Rodney, S. R. Energy Environ. Sci. 2010, 3, 12941301.

(8) Miller, J. R.; Simon, P. Science 2008, 321, 651-652.

(9) Zhang, H.; Cao, G. P.; Yang, Y. S. Energy Environ. Sci. 2009, 2, 932-939.

(10) Lee, S. W.; Gallant, B. M.; Byon, H. R.; Hammond, P. T.; Horn, Y. S. Energy Environ. Sci. 2011, 4, 1972-1979.

(11) Zhai, Y.; Dou, Y.; Zhao, D.; Fulvio, P. F.; Mayes, R. T.; Dai, S. Adv. Mater. 2011, 23, 4824-4850.

(12) Wang, Y.; Shi, Z. Q.; Huang, Y.; Ma, Y. F.; Wang, C. Y.; Chen, M. M.; Chen, Y. S. J. Phys. Chem. C 2009, 113, 13103-13107.

(13) Zhi, M. J.; Xiang, C. C.; Li, J. T.; Li, M.; Wu, N. Q. Nanoscale 2013, 5, 72-88.

(14) Chen, C. M.; Zhang, Q.; Huang, C. H.; Zhao, X. C.; Zhang, B. S.; Kong, Q. Q.; Wang, M. Z.; Yang, Y. G.; Cai, R.; Su, D. S. Chem. Commun. 2012, 48, 7149-7151.

(15) Gao, W.; Singh, N.; Song, L.; Liu, Z.; Reddy, L. M.; Ci, L. J.; Vajtai, R.; Zhang, Q.; Wei, B. Q.; Ajayan, P. M. Nat. Nanotechnol. 2011, 6, 496-500.

(16) Zhang, L. L.; Zhao, X.; Stoller, M. D.; Zhu, Y. W.; Ji, H. X.; Murali, S.; Wu, Y. P.; Perales, S.; Clevenger, B.; Ruoff, R. S. Nano Lett. 2012, 12, 1806-1812.

(17) Zhu, Y.; Murali, S.; Stoller, M. D.; Ganesh, K. J.; Cai, W.; Ferreira, P. J. Science 2011, 332, 1537-1541.

(18) Candelaria, S. L.; Shao, Y.; Zhou, W.; Li, X.; Xiao, J.; Zhang, J. G. Nano Energy 2012, 1, 195-220.

(19) Lee, D. H.; Kim, J. E.; Han, T. H.; Hwang, J. W.; Jeon, S.; Choi, S. Y. Adv. Mater. 2010, 22, 1247-1252. 
(20) Paul, R. K.; Ghazinejad, M.; Penchev, M.; Lin, J.; Ozkan, M.; Ozkan, C. S. Small 2010, 6, 2309-2313.

(21) Wang, Q.; Yan, J.; Wei, T.; Feng, J.; Ren, Y. M.; Fan, Z. J.;

Zhang, M. L.; Jing, X. Y. Carbon 2013, 60, 481-487.

(22) Dong, X. C.; Li, B.; Wei, A.; Cao, X. H.; Chan-Park, M. B.; Zhang, H. Carbon 2011, 49, 2944-2949.

(23) Wang, J.; Xu, Y. L.; Yan, F.; Zhu, J. B.; Wang, J. P. J. Power Sources 2011, 196, 2373-2378.

(24) Wang, H. L.; Hao, Q. L.; Yang, X. J.; Lu, L. D.; Wang, X. Nanoscale 2010, 2, 2164-2170.

(25) Moumita, K.; Awalendra, K. T.; Anil, K. B. ACS Appl. Mater. Interfaces 2013, 5, 8374-8386.

(26) Yu, P. P.; Li, Y.Z.; Yu, X. Z.; Zhao, X.; Wu, L. H.; Zhang, Q. H. Langmuir 2013, 29, 12051-12058.

(27) Wang, Y. F.; Yang, X. W.; Qiu, L.; Li, D. Energy Environ. Sci. 2013, 6, 477-481.

(28) Liao, Y. Z.; Zhang, C.; Zhang, Y.; Strong, V.; Tang, J. S.; Li, X. G.; Kalantar, Z. K.; Hoek, E. M.; Wang, K. L.; Kaner, R. B. Nano Lett. 2011, 11, 954-959.

(29) Fan, W.; Zhang, C.; Tjiu, W. W.; Pram, K. P.; He, C. B.; Liu, T. X. ACS Appl. Mater. Interfaces 2013, 5, 3382-3391.

(30) Miao, Y. E.; Fan, W.; Chen, D.; Liu, T. X. ACS Appl. Mater. Interfaces 2013, 5, 4423-4428.

(31) Liu, M. K.; Miao, Y. E.; Zhang, C.; Tiju, W. W.; Yang, Z. B.; Peng, H. S.; Liu, T. X. Nanoscale 2013, 5, 7312-7320.

(32) Wu, Q.; Xu, Y. X.; Yao, Z. Y.; Liu, A. R.; Shi, G. Q. ACS Nano 2010, 4, 1963-1970.

(33) Fan, H. S.; Wang, H.; Zhao, N.; Zhang, X. L.; Xu, J. J.

Mater.Chem. 2012, 22, 2774-2780.

(34) Cui, Q.; Zhou, J.; Shi, W.; Zhong, J.; Mi, H. Mater. Lett. 2013, 107, 141-143.

(35) Cong, H. P.; Ren, X. C.; Wang, P.; Yu, S. H. Energy Environ. Sci. 2013, 6, 1185-1191.

(36) Sung, H. C.; K-young, H. S.; Jyongsik, J. ACS Appl. Mater. Interfaces 2013, 5, 9186-9193.

(37) Yan, X. B.; Chen, J. T.; Yang, J.; Xue, Q. J.; Phiippe, M. ACS

Appl. Mater. Interfaces 2010, 2, 2521-2529.

(38) He, S. J.; Hu, X. W.; Chen, S. L.; Hu, H.; Hanif, M.; Hou, H. Q.

J. Mater. Chem. 2012, 22, 5114-5120.

(39) Cheng, Q.; Tang, J.; Ma, J.; Zhang, H.; Shinya, N.; Qin, L. C. J. Phys. Chem. C 2011, 115, 23584-23590.

(40) Stoller, M. D.; Park, S. J.; Zhu, Y. W.; An, J. H.; Ruoff, R. S. Nano Lett. 2008, 8, 3498-3502.

(41) Park, S.; Lee, K. S.; Bozoklu, G.; Cai, W. W.; Nguyen, S. T.; Ruoff, R. S. ACS Nano 2008, 2, 572-578.

(42) Xu, J. J.; Wang, K.; Zu, S. Z.; Han, B. H.; Wei, Z. X. ACS Nano 2010, 4, 5019-5026.

(43) Si, Y. C.; Samulski, E. T. Nano Lett. 2008, 8, 1679-1686.

(44) Yang, Q.; Shuai, L.; Pan, X. J. Biomacromolecules 2008, 9, 34223426.

(45) Tiwaria, A.; Kumar, R.; Prabaharan, M.; Pandey, R. R.; Kumari, P.; Chaturvedi, A.; Mishra, A. K. Polym. Adv. Technol. 2010, 21, 615620.

(46) Li, L.; Qin, Z. Y.; Liang, X.; Fan, Q. Q.; Lu, Y. Q.; Wu, W. H.; Zhu, M. F. J. Phys. Chem. C 2009, 113, 5502-5507.

(47) Hyeon-Jin, S.; Ki, K. K.; Anass, B.; Seon-Mi, Y.; Hyeon, K. P.; In-Sun, J.; Mei, H. J.; Hae-Kyung, J.; Jong, M. K.; Jae-Young, C.; Young, H. L. Adv. Funct. Mater. 2009, 19, 1987-1992.

(48) Zhong, M.; Song, Y.; Li, Y. F.; Ma, C.; Zhai, X. L.; Shi, J. L.; Guo, Q. G.; Liu, L. J. Power Sources 2012, 217, 6-12.

(49) Wang, W.; Guo, S. R.; Penchev, M.; Ruiz, I.; Bozhilov, K. N.; Yan, D.; Ozkan, M.; Ozkan, C. S. Nano Energy 2013, 2, 294-303.

(50) Xu, G.; Xu, D.; Zhang, J.; Wang, K.; Chen, Z.; Chen, J.; Xu, Q. J. Colloid Interface Sci. 2013, 411, 204-212.

(51) Cheng, Q.; Tang, J.; Shinya, N.; Qin, L. C. J. Power Sources 2013, 241, 423-428.

(52) Taeyoung, K.; Gyujin, J.; Seonmi, Y.; Kwang, S. S.; Rodney, S. R. ACS Nano 2013, 7, 6899-6905.
(53) Wang, Y. G.; Li, H. Q.; Xia, Y. Y. Adv. Mater. 2006, 18, 26192623. 